\title{
A New Method of Radar Signal Feature Parameter Assessment
}

\author{
Cheng Bolin ${ }^{1}$, Xu Jing ${ }^{2}$, Tang Yuwen ${ }^{1}$ \\ ${ }^{1}$ Air Force Early Warning Academy, Wuhan, China \\ ${ }^{2}$ No.94969 Unit the PLA, Shanghai, China
}

Email address:

dspbuilder_t@163.com (Tang Yuwen)

\section{To cite this article:}

Cheng Bolin, Xu Jing, Tang Yuwen. A New Method of Radar Signal Feature Parameter Assessment. Journal of Electrical and Electronic Engineering. Vol. 5, No. 2, 2017, pp. 33-37. doi: 10.11648/j.jeee.20170502.12

Received: January 18, 2017; Accepted: March 27, 2017; Published: April 6, 2017

\begin{abstract}
Aimed at the problem of radar signal feature parameter assessment in the process of sorting, a multi-index joint assessing method is proposed. This method uses complexity, separability and stability to assess the performance of feature parameters sorting. It overcomes the defects of traditional ways that cannot make an overall and scientific assessment by only using a single index. Meanwhile, it can get the optimal comprehensive score value under different application requirements. Its validity and good engineering utilization performance is proved by computer simulation.
\end{abstract}

Keywords: Radar Signal, Feature Extraction, Assessment

\section{Introduction}

With the continuous improvement of radar performance, the signal sorting technology based on the traditional five parameters is facing a severe test. Therefore, many researchers propose that through a certain mathematical transformation to extract the corresponding feature parameters from the intercepted radar signals. Then use these features to achieve the effective sorting of radar signals [1, 2, 3]. However, because of the complexity of the extraction of each feature parameter is different, and the degree of being affected by SNR and reflecting radar signal features is not the same, so the impact on sorting is different too. Therefore, the sorting performance of these features needs comprehensive assessment.

Nowadays, the accuracy of sorting is wildly used to assess the separable features of radar signal $[4,5]$. Because of the complex and changeable battlefield electromagnetic environment and the diversity and dynamic of battle application requirements, it is difficult to assess the performance of the feature parameters comprehensively and scientifically with only one index. To solve these problems, this paper proposes that using complexity, separability and stability to assess the performance of feature parameters. Each feature parameter can obtain a comprehensive score value under different application requirements, which can lay a foundation for the optimal parameter selection.

\section{Multi-Index Joint Assessing Method}

\subsection{Assessment Model}

In order to measure the sorting performance of a certain feature parameter, considering its complexity, separability and stability, and use these three indexes as the basic elements of a comprehensive assessment of the feature parameters. The basic meaning of the three indexes is as follows.

(1) Complexity. It indicates that the calculated amount to extract the feature parameters while the radar signal waveform data has been transformed.

(2) Separability. The feature parameters of radar signals with different modulation patterns and parameters are different, which can be used to sort the radar signals which do not belong to the same emitter source.

(3) Stability. Due to the complexity of the radar signal environment, the SNR of different pulses may be very different. Therefore, it is necessary that the feature parameters of radar signals do not fluctuate greatly with the fluctuation of SNR.

The feature parameters assessing model using the above three indexes as shown below. Where $\mathrm{S}_{\mathrm{i}}\left(0 \leq \mathrm{S}_{\mathrm{i}} \leq 1, \mathrm{i}=1,2,3\right)$ 
indicates the satisfaction degree of using the first $i$ index to assess the feature vector. Here 0 means total dissatisfaction, and 100 means complete satisfaction. Then, the satisfaction degree of several indexes is fused according to the importance of the index in the whole index system. Finally, the comprehensive satisfaction degree is $S_{a}$. As shown in the following formula:

$$
S_{a}=f\left(\mathbf{w}, \mathbf{s}_{i}\right)
$$

Where $\mathbf{w}$ is the weight vector, and $\mathbf{s}$ is the satisfaction vector.

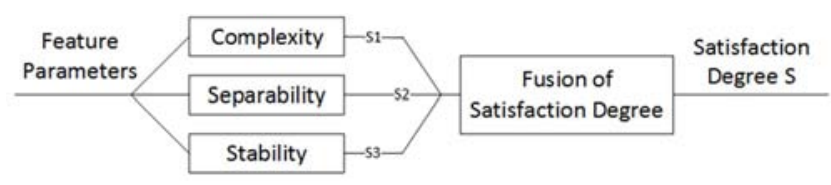

Figure 1. The model of feature parameters assessment.

\subsection{The Measurement of Assessment Indexes}

\subsubsection{The Measurement of Complexity}

The complexity of the feature parameters is reflected in the number of computer resources needed to extract the feature parameters, the more resources needed, the higher the complexity of the algorithm; On the contrary, the less the resources needed, the lower the complexity of the algorithm. The most important resources of computer are the time and space resources. Therefore, complexity can be divided into time complexity and space complexity. Because of the concept and computing method of time complexity and space complexity are similar, and its impact on time complexity is negligible when the space is adequate, so there mainly consider the time complexity.

The time taken to execute an algorithm is equal to the sum of the execution time of each statement in the algorithm. The execution time of each statement is equal to the number of times the statement is executed (i.e., the frequency) multiply by the time required to execute the statement once.

According to the increasing order of magnitude, common time complexity series is as follows: constant $O(1)$, logarithm order $O\left(\log _{2}^{n}\right)$, linear order $O(n)$, linear logarithm order $O\left(n \log _{2}^{n}\right)$, square order $O\left(n^{2}\right)$ 、 cubic order $O\left(n^{3}\right), \cdots$, k-power order $O\left(n^{k}\right)$, exponential order $O\left(2^{n}\right)$. Obviously, the exponential order $O\left(2^{n}\right)$ time complexity algorithm is very inefficient. It will be unusable when $n$ is slightly larger.

\subsubsection{The Measurement of Separability}

In the paper [1], a new method of category sorting is proposed, which overcomes the shortcomings of traditional methods. First of all, three definitions are given:

Definition 1: The category cohesion of the first $i$ class signals is:

$$
C_{i i}=\max _{k=1,2, \cdots, M_{i}^{q}}\left\{\left\|x_{i k}^{q}-E\left(X_{i}^{q}\right)\right\|\right\}
$$

In the formula, $q$ is the dimension of the feature vector, $M_{i}^{q}$ is the sample number of the class $i$ signals, $x_{i k}^{q}$ is the first $k$ sample vector of the $q$ dimensional feature of the class $i$ signals, $X_{i}^{q}=\left[x_{i 1}^{q}, x_{i 2}^{q}, \cdots, x_{i M_{i}^{q}}^{q}\right], E\left(X_{i}^{q}\right)$ is the expectation of $X_{i}^{q}$.

Definition 2: The distance of the class $i$ signals and the class $j$ signals is defined as:

$$
D_{i j}=\left\|E\left(X_{i}^{q}\right)-E\left(X_{j}^{q}\right)\right\|
$$

Where $E\left(X_{i}^{q}\right)$ and $E\left(X_{j}^{q}\right)$ are the expectation of $X_{i}^{q}$ and $X_{j}^{q}$.

Definition 3: The category sorting degree of the class $i$ signals and the class $j$ signals is defined as:

$$
S_{i j}=\frac{D_{i j}}{C_{i i}+C_{j j}}
$$

Where $D_{i j}$ is the distance of the class $i$ signals and the class $j$ signals, and $C_{i i}$ and $C_{j j}$ are the cohesion in category of the class $i$ signals and the class $j$ signals.

If there are $H$ kind of signals for sorting, according to the definition of category cohesion and category sorting given in the definition 1 and 3, the criterion function used to assess the quality of sorting degree of feature set is:

$$
f=\frac{2}{H(H-1)} \sum_{i=1}^{H-1} \sum_{j=i+1}^{H} \frac{S_{i j}}{q}
$$

Obviously, the higher the value of $f$ is, the higher the quality of the sorting degree of feature set is.

\subsubsection{The Measurement of Stability}

Feature parameters of different radar emitter signal has different stability, that is to say, the performance will be different under different SNR degree. There use signal factor variance analysis to study whether the noise have significant impact on the stability of feature parameters or not.

Assuming that the SNR has $m$ levels, writing for $S N R_{1}, \cdots, S N R_{m}$. Carrying out experiment $k$ times at each SNR level, and then a value will be obtained after each experiment, writing for this $C_{i j}$. It means the first $j$ experiment value in the first $i$ SNR level ( $i=1, \cdots, m ; j=1, \cdots, k)$.

In order to examine whether the SNR have significant impact on the experiment result or not, there regard $S N R_{1}, \cdots, S N R_{m}$ as $m$ normal population, and $C_{i j}$ is the first $j$ sample from the first $i$ population. Define the following variables: $S_{T}=\sum_{i=1}^{m} \sum_{j=1}^{k}\left(C_{i j}-\bar{C}\right)^{2}$ 


$$
\begin{gathered}
S_{e}=\sum_{i=1}^{m} \sum_{j=1}^{k}\left(C_{i j}-\bar{C}_{i}\right)^{2} \\
S_{A}=\sum_{i=1}^{m} \sum_{j=1}^{k}\left(\bar{C}_{i}-\bar{C}\right)^{2}=k \sum_{i=1}^{m}\left(\bar{C}_{i}-\bar{C}\right)^{2}
\end{gathered}
$$

where: $\bar{C}_{i}=\frac{1}{k} \sum_{j=1}^{k} C_{i j}, \bar{C}=\frac{1}{m} \sum_{i=1}^{m} \bar{C}_{i}=\frac{1}{m k} \sum_{i=1}^{m} \sum_{j=1}^{k} C_{i j}$

$S_{T}$ is called the sum of squares of total deviation, which is the sum of the difference between the observed value $C_{i j}$ and its total mean $\bar{C}$, which is the quantitative index of the degree of dispersion of all data. $S_{e}=\sum_{i=1}^{m} \sum_{j=1}^{k}\left(C_{i j}-\bar{C}_{i}\right)^{2}$ is the quadratic sum of the difference between the observed value and category mean value $\bar{C}_{i}$, which is called category quadratic sum or error quadratic sum. It reflects the random fluctuations of the samples within the group (at the same level). $S_{A}=k \sum_{i=1}^{m}\left(\bar{C}_{i}-\bar{C}\right)^{2}$ is the quadratic sum of difference between category mean value and total mean value, which is called interlock quadratic sum. It reflects the differences caused by different levels of each factor.

Which can be known from the sum of squares decomposition formula that the differences of observed values and total mean value can be regarded as two parts, which are the sum of squares intra the group (also called error sum of squares, it reflects the error caused by the random factors )and the sum of squares between the groups (the differences caused by different levels of each factor). Therefore, the ratio of $S_{A}$ to $S_{e}$ reflects the proportion of the two differences. If the ratio is greater, the difference of each level of the factors is significant. Therefore, we can define the statistical variable of the stability:

$$
F=\frac{S_{A} /(\mathrm{m}-1)}{S_{e} / m(\mathrm{k}-1)}
$$

$F$ can be used to test whether the effect of factors is significant. According to the threshold of $F_{\alpha}, F>F_{\alpha}$ indicates that the factors have significant impact on indexes, and the greater $F$ is the more significant the impacts are. If $F \leq F_{\alpha}$, it indicates that the factors don't have significant impact on indexes.

\subsection{The Standardization of Assessment Indexes}

The standardization of assessment indexes is to grade all the indexes related to feature parameters and transform each index into a "score" of polymerization by mathematical transformation. In order to facilitate the processing, there use "percentile system". Indexes can be divided into quantitative and qualitative. Separability and stability are qualitative indexes, while complexity is quantitative index. The standardization of quantitative indexes (also known as non dimensional) method is: mapping the index value to the upper and lower limits of 100 and 0 respectively. This type of transformation is a function from real number set to $[0,100]$, writing this $F(x): R \rightarrow[0,100]$ and call it the standardization function of index. The standardization of qualitative indexes is standardized by establishing an one-to-one mapping or qualitative rating scale.

\subsubsection{The Standardization of the Complexity}

The complexity index is a qualitative index, so it can't be processed by the normalization function. There quantify the level of the time complexity of each feature parameter, and establish a one-to-one mapping from index to score values. As

\begin{tabular}{|c|c|c|c|}
\hline Index & Logarithm order & Linear order & $\begin{array}{l}\text { Linear logarithm } \\
\text { order }\end{array}$ \\
\hline Score value & 100 & 95 & 90 \\
\hline Index & Square order & Cube order & $\begin{array}{l}\text { Quadruplicate } \\
\text { order }\end{array}$ \\
\hline Score value & 85 & 80 & 75 \\
\hline
\end{tabular}
shown in table 1 .

Table 1. Mapping from index to score value.

\subsubsection{The Standardization of the Separability}

The better the stability of feature parameter is, the higher accuracy is. Its growth trend is convex crescendo function. A convex crescendo function means that the function of this type is suitable for the situation that the score value increases with the increase of the actual value, and the tendency of gradually increasing becomes slower. The function form of index score value $Z_{i}$ as shown below:

$$
Z_{i}=\left\{\begin{array}{lrl}
0 & y_{i} \leq y_{i}^{\min } \\
100 \sin \left(\frac{y_{i}-y_{i}^{\min }}{y_{i}^{\max }-y_{i}^{\min }} \times \frac{\pi}{2}\right) & y_{i}^{\min }<y_{i}<y_{i}^{\max } \\
100 & y_{i} \geq y_{i}^{\max }
\end{array}\right.
$$

Where $Z_{i}$ is the score of $y_{i}$, while $y_{i}{ }^{\max }$ and $y_{i}{ }^{\min }$ are the satisfactory point and invalid point of $y_{i}$.

\subsubsection{The Standardization of the Stability}

The worse the stability of feature parameter is, the lower accuracy is. Its growth trend is convex decreasing function. A convex decreasing function means that the function of this type is suitable for the situation that the score value decreases with the decrease of the actual value, and the tendency of gradually decreasing becomes faster. The function form of index score value $Z_{i}$ as shown below:

$$
Z_{i}=\left\{\begin{array}{lrl}
0 & y_{i} \leq y_{i}^{\text {min }} \\
100 \sin \left(\frac{y_{i}^{\max }-y_{i}}{y_{i}^{\max }-y_{i}{ }^{\min }} \times \frac{\pi}{2}\right) & y_{i}^{\min }<y_{i}<y_{i}^{\text {max }} \\
100 & y_{i} \geq y_{i}^{\max }
\end{array}\right.
$$

\subsection{Multi-Index Comprehensive Assessment}

As can be seen from the above analysis that the final assessment of value should fuse the assessment result of the complexity, separability and stability. This paper uses multi-index weighted calculation method for each index. 
Through given a certain weight to each index, a comprehensive score $f$ of each feature parameter values can be obtained. As shown in the following formula:

$$
f=f_{1} \varepsilon_{1}+f_{2} \varepsilon_{2}+f_{3} \varepsilon_{3}
$$

Where $f_{1}, f_{2}, f_{3}$ are the score values of complexity, separability and stability and $\varepsilon_{1}, \varepsilon_{2}, \varepsilon_{3}$ are the weight of each index. The weights are determined by the actual application requirements. As can be seen from the formula (9), when the weight of each index is changed, the comprehensive score will change, which means the comprehensive score of each feature parameter value is dynamic. It will change along with the changing of signal environment and the application demand, which is consistent with the current operational requirements.

\section{The Simulation Analysis}

In order to better illustrate the assessment method of this article, there use five kinds of radar signal features from the current literatures and the previous studies to verify it. They are fuzzy function features, resemblance coefficient, box dimension features, entropy features and bispectrum features [1, 6-10]. Through the above analysis, the score value parameters of each index of five features can be obtained. Details as shown in the following table.

Table 2. The score value of each index of feature parameters.

\begin{tabular}{llll}
\hline Features & Complexity & Separability & Stability \\
\hline Fuzzy function features & 85 & 94.4 & 96.5 \\
Similar coefficient & 95 & 87.2 & 89.3 \\
Box dimension features & 95 & 90.8 & 90.9 \\
Entropy features & 95 & 89.3 & 91.3 \\
Bispectrum features & 85 & 95.5 & 98.7 \\
\hline
\end{tabular}

As can be seen from the table above, each index from different feature parameters have different performance, they all have advantages and disadvantages. For example, similar coefficient, box dimension feature and entropy feature own the best complexity, while bispectrum features possess the best separability and stability. Then there use two specific cases to analyze and verify it.

Case 1: there are four types of radar signal contained in the received radar signal sequence, each signal sequence of different radar contains 1000 signals, a total of 4000. Signal forms are CW, LFM, FSK and BPSK. Random noise is added to each signal, the noise size is $15-20 \mathrm{~dB}$. There assume that the score values of complexity, separability and stability are required to be 60,90 and 60 respectively when doing sorting processing. As can be seen from the score values, the primary demand is the separability, that is to say, a higher accuracy is needed when use feature parameters to sort, while the requirement of complexity and stability is lower than separability. We can obtain $\varepsilon_{1}=0.286, \varepsilon_{2}=0.428, \varepsilon_{3}=0.286$ under the application requirements. After calculating from the formula above, the comprehensive score values of fuzzy function features, box dimension features and bispectrum features respectively as 92.31, 92.03, 93.41. Therefore, under the current application requirements, bispectrum features have the best comprehensive performance. In order to further verify the validity and rationality of this assessment method proposed in this paper, respectively using the five features to sort signal sequence, to visually reflect the sorting performance of five feature parameters in the current application demand, sorting results shown in the table below. It can be seen that the sorting accuracy of bispectrum is the highest, which can reach $99 \%$, and it meets the current applications better than other parameters.

Table 3. The sorting results of fuzzy function features and bispectrum features.

\begin{tabular}{llll}
\hline Index & $\begin{array}{l}\text { Fuzzy function } \\
\text { features }\end{array}$ & $\begin{array}{l}\text { Similar } \\
\text { coefficient }\end{array}$ & $\begin{array}{l}\text { Box dimension } \\
\text { features }\end{array}$ \\
\hline Sorting time/s & 84 & 6.9 & 7 \\
Sorting accuracy rate & 0.989 & 0.962 & 0.984 \\
Index & Entropy features & $\begin{array}{l}\text { Bispectrum } \\
\text { features }\end{array}$ & \\
Sorting time/s & 7.1 & 87 & \\
Sorting accuracy rate & 0.971 & 0.99 & \\
\hline
\end{tabular}

Case 2: there are four types of radar signal contained in the received radar signal sequence, each signal sequence of different radar contains 1000 signals, a total of 4000 . Signal forms are CW, LFM, FSK and BPSK. Random noise is added to each signal, the noise size is $0-5 \mathrm{~dB}$. There assume that the score values of complexity, separability and stability are required to be 90,70 and 90 respectively when doing sorting processing. As can be seen from the score values, the primary demands are the complexity and stability, that is to say, a higher accuracy is needed when use feature parameters to sort, while the requirement of complexity and stability is lower than separability. We can obtain $\varepsilon_{1}=0.36, \varepsilon_{2}=0.28, \varepsilon_{3}=0.36$ under the application requirements. After calculating from the formula above, the comprehensive score values of box dimension features and bispectrum features respectively as 92.348, 92.072. Therefore, under the current application requirements, box dimension features have the best comprehensive performance. In order to further verify the validity and rationality of this assessment method proposed in this paper, respectively using the five features to sort signal sequence, to visually reflect the sorting performance of five feature parameters in the current application demand, sorting results shown in the table below. It can be seen that although the sorting accuracy of fuzzy function features and bispectrum features are the highest, but their sorting time can hardly meet the current processing requirement, while the time of box dimension feature is the shortest and its accuracy is ideal, which can reach to $91.3 \%$ and meet the application requirement in need.

Table 4. The sorting results of fuzzy function features and bispectrum features.

\begin{tabular}{llll}
\hline Index & $\begin{array}{l}\text { Fuzzy function } \\
\text { features }\end{array}$ & $\begin{array}{l}\text { Similar } \\
\text { coefficient }\end{array}$ & $\begin{array}{l}\text { Box dimension } \\
\text { features }\end{array}$ \\
\hline Sorting time/s & 84 & 6.9 & 7 \\
Sorting accuracy rate & 0.931 & 0.885 & 0.913 \\
Index & Entropy features & $\begin{array}{l}\text { Bispectru } \\
\text { m features }\end{array}$ \\
Sorting time/s & 7.1 & 87 & \\
Sorting accuracy rate & 0.908 & 0.94 & \\
\hline
\end{tabular}


These results indicates that the assessment indexes and quantitative method proposed in this paper is accurate and effective. They can choose the optimal performance parameters under different application requirements.

\section{Conclusion}

The radar emitter signal sorting and recognition is one of the important part of electronic warfare, and the performance of sorting and recognition is greatly affected by the feature parameter elected. At present, the accuracy of sorting and recognition is widely used to assess the performance of parameters, but because of the complex and changeable battlefield electromagnetic environment and the diversity and dynamic of battle application requirements, it is difficult to assess the performance of the feature parameters comprehensively and scientifically with only one index. In order to solve the problems above, this article proposes that to use complexity, separability and stability to assess the feature parameters under different application requirements, which can lay a foundation for the optimal parameter selection.

\section{References}

[1] Zhang Gexiang, "Intelligent Recognition Methods for Radar Emitter Signals," D. Southwest Jiaotong University, Ph. D. Thesis, 2005.

[2] Jun Han, Ming-hao He, Yan Mao, Ming-qiu Ren. A New Method for Recognizing Radar Radiating-source. J. ICWAPR'2007, pp. 1665-1668.
[3] Guo Lirong, He Minghao, Yu Chunlai, Wang Bingqie, "Radar Emitter Signal Sorting Based on Pulse Phase Linearity," Journal of CABT, vol.9 No.1, Feb. 2014, pp.50-54.

[4] Guo Qiang, Wang Changhong, Li Zheng, "Support Vector Clustering and Type-Entropy Based Radar Signal Sorting Method," Journal of Xi' an Jiaotong University, vol.44, 2010, pp.63-67.

[5] Wang Shiqiang, Zhang Dengfu, Bi Duyan, Yong Xiaoju, "Multi-parameter Radar Singal Sorting Method Based on Fast Support Vector Clustering and Similitude Entropy," Journal of Electronics \& Information technology, vol.33 No.11, 2011, pp.2735-2741.

[6] Han Jun, He Minghao, Zhu Yuanqing, Mao Yan, "Sorting of Radar Emitter Based on Milti-Parameters, Journal of Data Acquisition\&Processing," vol. 24 No.1, 2009, pp.91-94.

[7] CHEN Changxiao, HE Minghao, XU Jing, HAN Jun, "A New Method for Sorting Unknown Radar Emitter Signal”, Chinese Journal of Electronics, vol.23 No.3, 2014, pp.499-502.

[8] Gexiang Zhang, Haina Rong, Laizhao Hu, Weidong Jin, "Entropy feature extraction approach of radar emitter signals", Proceedings of International Conference on Intelligent Mechatronics and Automation, 2004, pp.621-625.

[9] YONG Xiao-ju, ZHANG Deng-fu, WANG Shi-qiang, “A new method of sorting radar emitter signals", Modern Defence Technology, Vol.39, No.3, 2011 pp.148-151.

[10] LIANG Hua-dong, XU Qing, “Application of entropy features to radar signal sorting", Journal of Air Force Early Warning Academy, Vol.29, No.1, 2015, pp.7-12. 